\title{
Sub-cycle optical phase control of nanotunnelling in the single-electron regime
}

\author{
Tobias Rybka, Markus Ludwig, Michael F. Schmalz, Vanessa Knittel, Daniele Brida` \\ and Alfred Leitenstorfer*
}

The high peak electric fields provided by single-cycle light pulses can be harnessed to manipulate and control charge motion in solid-state systems, resulting in electron emission out of metals and semiconductors ${ }^{1-6}$ or high harmonics generation in dielectrics ${ }^{7,8}$. These processes are of a non-perturbative character and require precise reproducibility of the electric-field profile ${ }^{9-14}$. Here, we vary the carrier-envelope phase of 6-fs-long near-infrared pulses with $\mathrm{pJ}$-level energy to control electronic transport in a laterally confined nanoantenna with an $8 \mathrm{~nm}$ gap. Peak current densities of $50 \mathrm{MA} \mathrm{cm}^{-2}$ are achieved, corresponding to the transfer of individual electrons in a half-cycle period of $\mathbf{2} \mathrm{fs}$. The observed behaviours are made possible by the strong distortion of the effective tunnelling barrier due to the extreme electric fields that the nanostructure provides and sustains under sub-cycle optical biasing. Operating at room temperature and in a standard atmosphere, the performed experiments demonstrate a robust class of nanoelectronic switches gated by phase-locked optical transients of minute energy content.

Present-day high-frequency devices operate in the microwave range, but direct control of electron flow by the electric-field profile of few-cycle optical pulses has recently been demonstrated ${ }^{12,15}$. These experiments were based on strong perturbation of the electronic system in a dielectric material, resulting in a large incoherent background current. Nanoscale confinement of the region biased by the optical transient might avoid a significant influence of nonlinear conductivity in an insulating substrate and result in purely coherent tunnelling currents ${ }^{16}$ that may be controlled by the precise shape of the electric field cycles. Our approach to reaching this goal is illustrated in the upper part of Fig. 1a. A single-cycle near-infrared pulse (left) is focused to a nanoscale junction of an electronic circuit (centre) with nonlinear and antisymmetric current-voltage $(I-V)$ characteristics (right). An effective bias then arises when the exciting electric field is cosine-shaped (blue transient and blue dot in the $I-V$ scheme) because its extremum occurs only for one polarity. Consequently, the symmetry of electronic transport is broken, even when integrating over the entire transient, and a net tunnelling current of electrons results through the potential barrier represented by the free-space gap (Fig. 1b,c). Our experiment favours tunnelling over other phenomena such as multiphoton ionization ${ }^{4}$ by operating with ultrashort pulses and extreme nanoconfinement of the electric field. No background current exists when the control field is sineshaped (red transient and dot in the $I-V$ characteristics, respectively), because positive and negative polarities now have precisely opposite effects. Consequently, the total current amplitude and direction may be set by varying the carrier-envelope phase (CEP), $\Delta \varphi_{\mathrm{CEP}}$, of the pulse. Owing to the single-cycle pulse duration, nanoscale constriction and field enhancement of the antenna device, quantum tunnelling may be controlled with optical pulse energies at the picojoule scale a

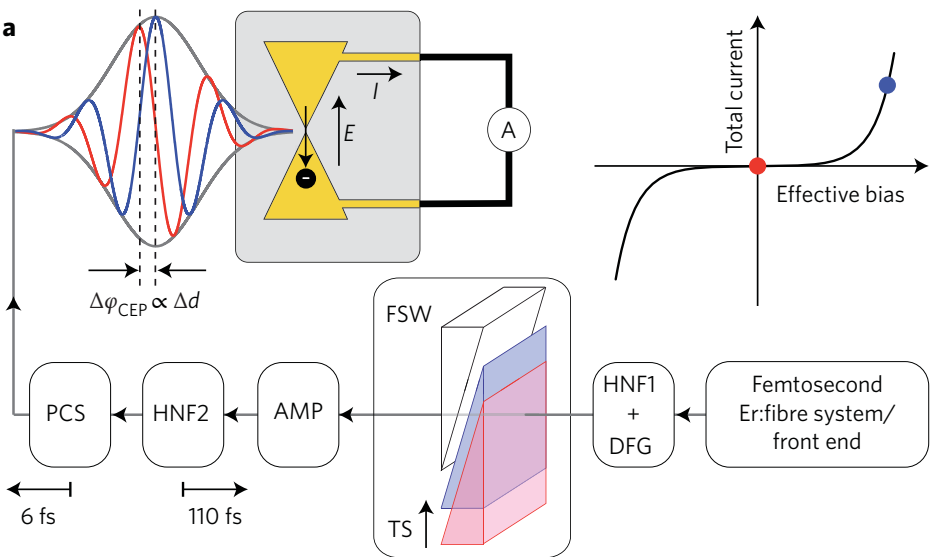

b

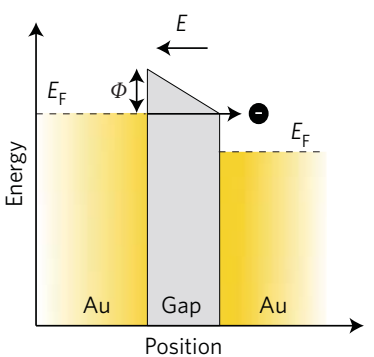

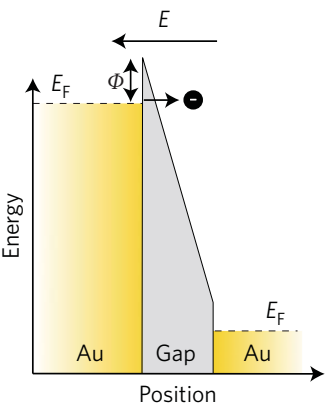

Figure 1 | Carrier-envelope optical phase control of tunnelling currents in a nanodevice. a, Top: sketch of single-cycle optical transients (left) triggering electron tunnelling in a nanoantenna electric circuit (centre) with nonlinear and antisymmetric current-voltage characteristics (right). Colours of transients and dots denote extremal biasing conditions and integrated current (red, sine-like pulses; blue, cosine-like pulses). I, current; A, ampere meter; $E$, electric field. Bottom: set-up consisting of femtosecond front end, highly nonlinear fibres (HNF1, HNF2), a difference frequency generation (DFG) source and Er:fibre amplifier (AMP). PCS, synthesis of 1.4-cycle pulses. The optical thickness $\Delta d$ of a pair of fused-silica wedges (FSW) is set by a translation stage (TS) to control the carrier-envelope phase $\Delta \varphi_{\mathrm{CEP}}$ before the pulse duration is reduced from 110 to $6 \mathrm{fs}$. $\mathbf{b}$, Spatial band structure at the dielectric gap under moderate stationary bias. c, Sketch of the transient conditions during a strong optical pulse. $E_{\mathrm{F}}$, Fermi energy; $\Phi$, work function of Au. 
a

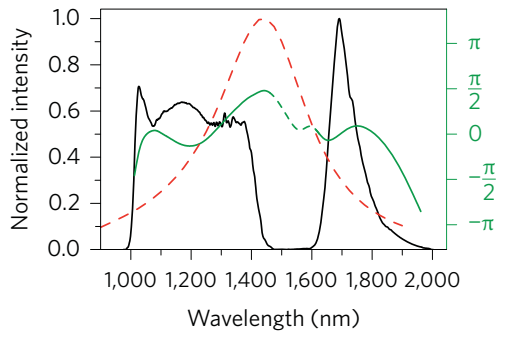

b

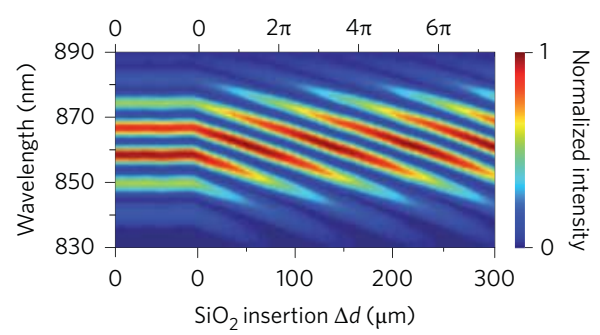

c

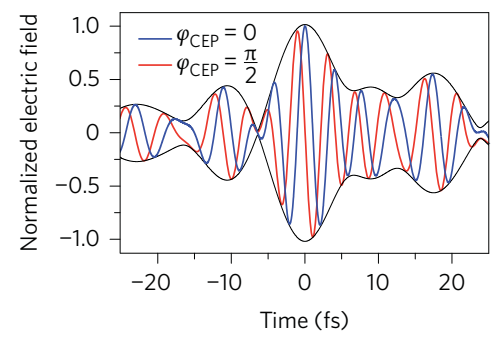

Figure 2 | Characterization of phase-locked optical control transients. a, Spectral intensity profile (black line) and phase (green line) of the near-infrared biasing pulses compared to the calculated resonance profile of the plasmonic nanoantenna (red dashed line). $\mathbf{b}, f$-to- $2 f$ spectrogram of the pulses as a function of position of the fused-silica wedges. The intensity of the interferogram is colour-coded versus wavelength. $\Delta d=84 \mu \mathrm{m}$ corresponds to a shift of $\Delta \varphi_{\text {CEP }}$ by $2 \pi$. c, Extracted electric-field profile for a cosine-like $\left(\varphi_{\text {CEP }}=0\right.$, blue line $)$ and sine-like $\left(\varphi_{\text {CEP }}=\pi / 2\right.$, red line $)$ phase of the control pulse, as obtained by a Fourier transform of the complex frequency spectra.
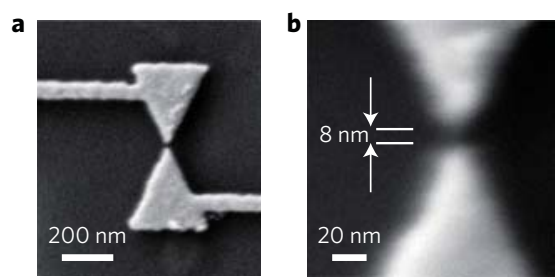

c Field enhancement $\begin{array}{llll}35 & 30 & 25 & 20\end{array}$

d

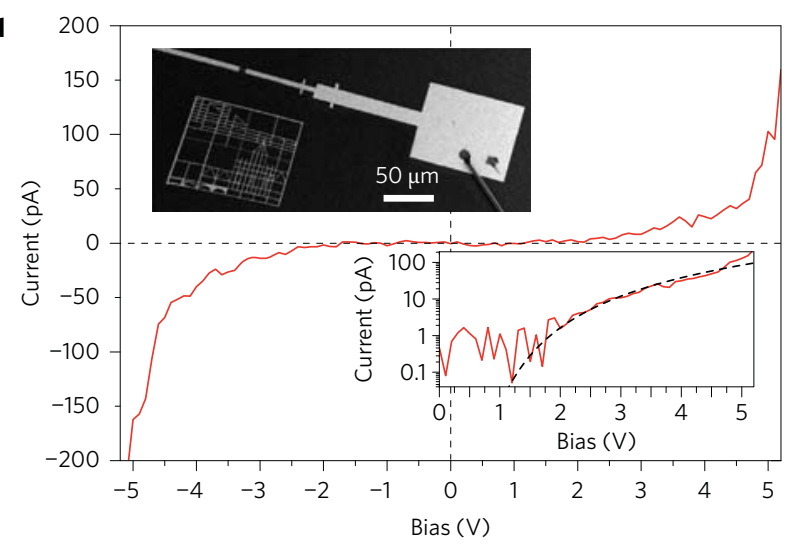

Figure 3 | Stationary characterization of the nanocircuit. a, SEM of an optical bowtie antenna with electric contacts. $\mathbf{b}$, Detailed view of the feedgap region. $\mathbf{c}$, Relative field enhancement calculated in the free space (colour map) between the antenna tips (black sections). White areas correspond to regions where our classical simulation would yield unrealistic results due to neglecting the evanescent character of the surface electronic wavefunctions. d, Stationary current-voltage characteristics of the nanoantenna circuit (red lines). Lower inset: dependence plotted on a logarithmic scale with a Fowler-Nordheim fit (black dashed line). Upper inset: SEM of macroscopic contacts leading to the nanodevice.

$\left(1 \mathrm{pJ}=10^{-12} \mathrm{~J}\right)$, which are available from compact laser systems based on telecom technology. Such a source is sketched in the lower part of Fig. 1a. A femtosecond Er:fibre front end delivers a pulse train at a wavelength of $1.55 \mu \mathrm{m}$ and repetition rate of $80 \mathrm{MHz}$. A highly nonlinear fibre (HNF) provides a coherent supercontinuum ${ }^{17}$ that supports intrapulse difference frequency mixing. This process generates a transient with passively locked CEP (ref. 17) that can be precisely set by varying the insertion $\Delta d$ of a pair of fused-silica wedges before re-amplification. Note that CEP control occurs at a pulse duration of $110 \mathrm{fs}$ (Fig. 1a), thus without distorting the envelope of the final single-cycle pulse, which is generated in a second HNF.

The resulting control transients are analysed in Fig. 2. Their octave-spanning intensity spectrum (black line in Fig. 2a) coherently combines ${ }^{18}$ a broadband dispersive wave centred at a wavelength of $1,200 \mathrm{~nm}$ and a soliton around 1,700 nm. The spectral phase (green line in Fig. 2a) was measured with two-dimensional shearing interferometry ${ }^{19}$ (2DSI). CEP stability and control was analysed from the $f$-to- $2 f$ spectral beats ${ }^{20}$ in Fig. 2b. Initially, the silica wedges were left untouched. This interval demonstrates a root-mean-square (r.m.s.) stability of $\Delta \varphi_{\mathrm{CEP}}$ better than $0.02 \mathrm{rad}$ over several minutes. Subsequently, the material insertion $\Delta d$ was varied up to $300 \mu \mathrm{m}$, resulting in a continuous change in the CEP. The extreme limits of cosine-like $\left(\varphi_{\mathrm{CEP}}=0\right.$, blue line in Fig. $\left.2 \mathrm{c}\right)$ and sine-like $\left(\varphi_{\text {CEP }}=\pi / 2\right.$, red line in Fig. $\left.2 c\right)$ electric-field transients were extracted by Fourier transforms of their spectral amplitude and phase (Fig. 2a). The full-width at half-maximum of the intensity of the pulses was equal to $6.3 \mathrm{fs}$, corresponding to 1.4 cycles at the central wavelength of $1,325 \mathrm{~nm}$.

The nanodevice controlled by these transients is characterized in Fig. 3. A scanning electron micrograph (SEM) of an optical antenna with lateral electric contacts ${ }^{21}$ is presented in Fig. 3a. The close-up view of the device in Fig. $3 \mathrm{~b}$ highlights a narrow feedgap of $\sim 8 \mathrm{~nm}$. Significant field enhancement ${ }^{22}$ was confirmed by numerical simulations based on a classical boundary element method ${ }^{23}$ (Fig. 3c). The strong radiation damping of the bowtie design results in a broadband amplitude response ${ }^{24}$ (red dashed line in Fig. 2a) and the antenna resonance is designed to be centred at the intensity gap between the soliton and dispersive wave. Together, these aspects preserve the few-cycle character of the enhanced field. The $I-V$ characteristics of the device under stationary bias are presented in Fig. 3d together with a global SEM overview showing the lead structure all the way to one of the bond pads that connect to a current amplifier (upper inset). The current is highly nonlinear and antisymmetric with respect to positive and negative bias (red line in Fig. 3d). As seen on a logarithmic scale in the lower inset of Fig. 3d, the dependence fits a Fowler-Nordheim ${ }^{25}$ behaviour well (black dashed line), as expected for a tunnelling junction until approaching a bias equivalent to the work function $\Phi$ of $\mathrm{Au}$ of $5.4 \mathrm{eV}$. The typical parameters of the electronic system under such moderate conditions are sketched in Fig. $1 \mathrm{~b}$.

In our experiments, we exposed a single contacted nanoantenna with an $8 \mathrm{~nm}$ gap to the CEP-locked single-cycle pulses focused by a Cassegrain objective with 0.65 numerical aperture. The optical electric field $E$ is polarized along the longitudinal antenna axis (Fig. 1a). We expect the transients to trigger tunnelling current spikes at the repetition rate of the laser when the CEP is set to a cosine-like shape. Laser excitation was modulated by a mechanical chopper operating at $632 \mathrm{~Hz}$, and electronic readout was performed with a transimpedance amplifier in combination with a lock-in detection scheme. We were indeed able to control the direction of the current by shifting the CEP of the driving pulses. Biasing with an average optical power of $6.5 \mathrm{~mW}$, corresponding to $80 \mathrm{pJ}$ of energy per pulse focused to a spot diameter of $1 \mu \mathrm{m}$, resulted in 


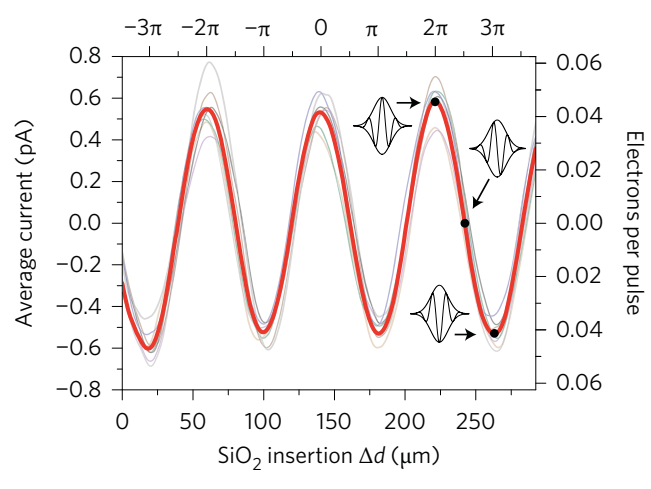

b

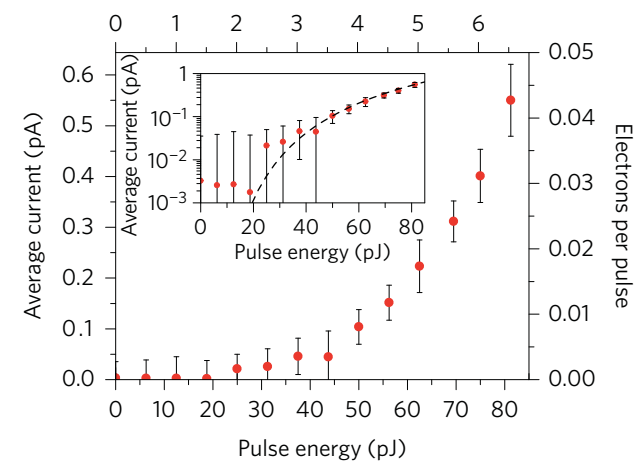

c

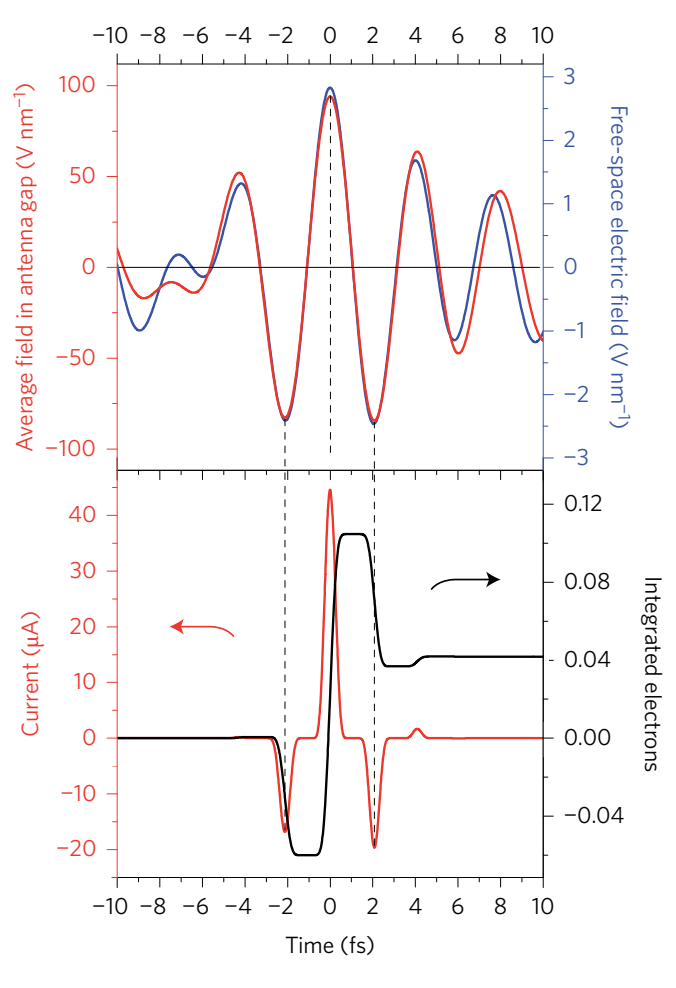

Figure 4 | Carrier-envelope optical control of sub-cycle tunnelling in the nanoantenna. a, Total current over the nanoantenna versus fused-silica insertion $\Delta d$ controlling the CEP of the excitation pulse at an optical power of $6.5 \mathrm{~mW}$. Black symbols indicate the fundamental symmetry of the phase-locked transients. The red line represents an average over eight subsequent measurements (thin grey lines). Each data point results from 50 million driving pulses. b, Current for a cosine-like transient versus optical pulse energy. Inset: the same data set on a logarithmic scale with least-squares fit (black dashed line). c, Top: electric-field transient in free space (blue line) with $\varphi_{\mathrm{CEP}}=0$ for maximal current injection. The red line represents the optical field enhanced and shaped by the plasmonic resonance at the nanoantenna gap. Its duration corresponds to 1.6 cycles of the carrier frequency. Bottom: instantaneous tunnelling current (red line) and accumulated electronic charge per pulse (black line).

total currents up to $0.6 \mathrm{pA}$. Under those conditions, the peak electric field in free space around the nanoantenna amounts to $2.9 \mathrm{~V} \mathrm{~nm}^{-1}$. The total current measured as a function of CEP, controlled by the wedge insertion, is presented in Fig. 4a. The red line represents an average over eight successive scans taken at a lock-in bandwidth of $1.6 \mathrm{~Hz}$. Independent acquisitions are depicted in grey in the background, underlining the extreme accuracy of the sinusoidal dependence of the total current on $\varphi_{\text {CEP }}$ and its reproducibility on a timescale of several minutes. We work under statistical conditions of transport where only one single electron on average is tunnelling every 20 pulses when the driving transient is cosine-shaped (Fig. 4a). The extreme precision of the spatiotemporal confinement, CEP control and sensitivity of our set-up allows us to study such a dilute regime, even at room temperature.

A strongly nonlinear dependence of the peak current on the optical excitation power is depicted by red dots in Fig. 4b. The extreme electric fields applied optically to the nanodevice by the single-cycle transient drive electron transport into a fundamentally different range (Fig. 1c) as compared to the quasi-static current corresponding to the conditions sketched in Fig. $1 \mathrm{~b}$. In the optical case, the free-space bias applied to the antenna gap approaches $25 \mathrm{~V}$. By considering a 35-fold enhancement of the antenna (Fig. 3c), we estimate an electric field up to $100 \mathrm{~V} \mathrm{~nm}^{-1}$ in the gap region, corresponding to a Keldysh parameter of $\sim 0.05$. Such strong bias is still non-disruptive, because it is applied only for a temporal duration of less than a half cycle of the optical field, which amounts to $2 \mathrm{fs}$ in our experiment. Under this extreme perturbation, the effective tunnelling barrier shrinks to a width that might ultimately approach the typical extension of the evanescent part of the surface electronic wavefunctions of the metal extending into free space ${ }^{26-28}$ (Fig. 1c). Electrons from the entire Fermi sea may be involved, with the ones close to the top having the highest probability of contributing. In a semiclassical picture, carriers tunnel into the gap, and the total probability of traversing the entire space all the way to the opposite contact might then be dominated by field-driven ballistic propagation. We estimate a typical transit time needed to bridge the $8 \mathrm{~nm}$ gap of 900 as under the highest biasing conditions outlined by Fig. 4a,b. This value is to be understood as an average, because some electrons that originate from the spatial tail of the wavefunctions inside the gap may be transferred faster, while the ones emitted after the field maximum accumulate less acceleration. It should be noted that the transport process is fully coherent with the driving field, because all the physical steps that establish the ultrafast current are in phase with each optical half cycle of the light pulse. Analysis of the instantaneous tunnelling current necessitates knowledge of the time-dependent field as well as the nonlinear $I-V$ dependence that applies under optical bias. A conservative assessment based on our experimental findings works as follows. We consider the cosine-like field transient measured in Fig. 2c and the retardation effect caused by the plasmonic resonance. The fast damping time of the bowtie antenna together with the partially off-resonant excitation ensures a minimum difference between the free-space driving field (blue line in the upper part of Fig. 4c) and the transient bias in the plasmonic gap (red line). A Fowler-Nordheim function is adopted to locally approximate the nonlinear response. We then calculate the total transferred 
charge by integrating over time. The parameters are adjusted to fit the dependence of the maximum average current on pump Fig. 4c shows the instantaneous current (red line in the lower part) that results under maximum optical bias. Only the main three extrema of the driving field contribute significantly, and the central one dominates, eventually leading to a finite total current. The net charge transferred per pulse (black line in the lower Our measurement of 0.043 electrons at a pulse energy of $80 \mathrm{pJ}$ (Fig. 4b) fixes the limit achieved at late times after the transient in Fig. 4c. Interestingly, this estimate corresponds to tunnelling of more than 0.15 electrons per central half cycle on average, as evidenced by the largest step in the black graph in the lower part of Fig. 4c. Consequently, a clear route to increase the cumulated tunnelling probability requires further reduction of the driving pulse duration to minimize the contribution of the cycles adjacent to the global maximum of the optical field. Note that our evaluation is robust with respect to the specific assumption on the functional form of the nonlinear $I-V$ characteristics. We tested several other options of exponential and high-order polynomial fits to the data in Fig. 4b, and found only minor effects on the assessment of the microscopic peak current. Instead, low-order polynomials fail in reproducing the experimental data, underlining the necessity of a strongly superlinear response. Clearly, any microscopic description of our experiment calls for a future theory of tunnelling and quantum transport over the free-space nanogap under extreme optical bias. In particular, it will be interesting to consider the spatiotemporal dynamic aspects intrinsic to a problem that is inherently nonlocal.

In conclusion, we have demonstrated precise CEP control of the electronic current over a laterally constricted tunnelling gap with dimensions of a few nanometres. Analysis of the data yields peak values up to $45 \mu \mathrm{A}$. Combined with an effective cross-section of $\sim 80 \mathrm{~nm}^{2}$ (Fig. 3b,c), we obtain sub-cycle current densities exceeding $50 \mathrm{MA} \mathrm{cm}^{-2}$, driven at room temperature and without damaging the device. More than 0.1 electrons are found to tunnel during the most prominent half cycle of a few-femtosecond phase-locked driving pulse with energy as low as $80 \mathrm{pJ}$. These results represent an encouraging step towards a new class of electronic nanodevices that operate with extremely high bandwidth and exploit advanced concepts like Coulomb blockade or resonant tunnelling without the need for cryogenic cooling. Promising directions include shrinking the dimensions of the gap towards the molecular regime or placing a strongly confined semiconductor structure into it. For example, by positioning a colloidal quantum dot between both metal electrodes, it may be possible to observe the onset of individual tunnelling channels, leading to deterministic transport. Studying quantum transport on truly elementary timescales is attractive, for example because electron-electron interactions may become extremely strong under such transient conditions where Coulomb screening is not yet established ${ }^{29}$. Our experiment also motivates intersection between attosecond optics and quantum plasmonics. pulse energy (dashed line in the inset of Fig. 4b). As an example, part of Fig. 4c) is obtained by integrating the sub-cycle current. theoretical efforts to describe mesoscopic electron systems at the

3. Swanwick, M. E. et al. Nanostructured ultrafast silicon-tip optical field-emitter arrays. Nano Lett. 14, 5035-5043 (2014).

4. Wimmer, L. et al. Terahertz control of nanotip photoemission. Nat. Phys. 10, 432-436 (2014).

5. Herink, G., Wimmer, L. \& Ropers, C. Field emission at terahertz frequencies ACtunneling and ultrafast carrier dynamics. New J. Phys. 16, 123005 (2014).

6. Vogelsang, J. et al. Ultrafast electron emission from a sharp metal nanotaper driven by adiabatic nanofocusing of surface plasmons. Nano Lett. 15, 4685-4691 (2015).

7. Hohenleutner, M. et al. Real-time observation of interfering crystal electrons in high-harmonic generation. Nature 523, 572-575 (2015).

8. Luu, T. T. et al. Extreme ultraviolet high-harmonic spectroscopy of solids. Nature 521, 498-502 (2015).

9. Lemell, C., Tong, X.-M., Krausz, F. \& Burgdörfer, J. Electron emission from metal surfaces by ultrashort pulses determination of the carrier-envelope phase. Phys. Rev. Lett. 90, 076403 (2003).

10. Dombi, P., Krausz, F. \& Farkas, G. Ultrafast dynamics and carrier-envelope phase sensitivity of multiphoton photoemission from metal surfaces. J. Mod. Opt. 53, 163-172 (2006)

11. Krüger, M., Schenk, M. \& Hommelhoff, P. Attosecond control of electrons emitted from a nanoscale metal tip. Nature 475, 78-81 (2011)

12. Schiffrin, A. et al. Optical-field-induced current in dielectrics. Nature 493 70-74 (2012).

13. Piglosiewicz, B. et al. Carrier-envelope phase effects on the strong-field photoemission of electrons from metallic nanostructures. Nat. Photon. 8 , 37-42 (2013).

14. Neppl, S. et al. Direct observation of electron propagation and dielectric screening on the atomic length scale. Nature 517, 342-346 (2015).

15. Paasch-Colberg, T. et al. Solid-state light-phase detector. Nat. Photon. 8, 214-218 (2014)

16. Cocker, T. L. et al. An ultrafast terahertz scanning tunnelling microscope. Nat. Photon. 7, 620-625 (2013).

17. Brida, D., Krauss, G., Sell, A. \& Leitenstorfer, A. Ultrabroadband Er:fiber lasers. Laser Photon. Rev. 8, 409-428 (2014).

18. Krauss, G. et al. Synthesis of a single cycle of light with compact erbium-doped fibre technology. Nat. Photon. 4, 33-36 (2010).

19. Birge, J. R., Ell, R. \& Kärtner, F. X. Two-dimensional spectral shearing interferometry for few-cycle pulse characterization. Opt. Lett. 31, 2063-2065 (2006)

20. Kakehata, M. et al. Single-shot measurement of carrier-envelope phase changes by spectral interferometry. Opt. Lett. 26, 1436-1438 (2001).

21. Kern, J. et al. Electrically driven optical antennas. Nat. Photon. 9, 582-586 (2015).

22. Mühlschlegel, P., Eisler, H.-J., Martin, O. J. F., Hecht, B. \& Pohl, D. W. Resonant optical antennas. Science 308, 1607-1609 (2005).

23. Hohenester, U. \& Trügler, A. MNPBEM-a Matlab toolbox for the simulation of plasmonic nanoparticles. Comput. Phys. Commun. 183, 370-381 (2012).

24. Hanke, T. et al. Tailoring spatiotemporal light confinement in single plasmonic nanoantennas. Nano Lett. 12, 992-996 (2012).

25. Fowler, R. H. \& Nordheim, L. Electron emission in intense electric fields. Proc. R. Soc. Lond. A 119, 173-181 (1928).

26. Savage, K. et al. Revealing the quantum regime in tunnelling plasmonics. Nature 491, 574-577 (2012).

27. Esteban, R. et al. A classical treatment of optical tunneling in plasmonic gaps: extending the quantum corrected model to practical situations. Faraday Discuss. 178, 151-183 (2015).

28. Wan, Y., Wubs, M. \& Mortensen, N. A. Projected dipole model for quantum plasmonics. Phys. Rev. Lett. 115, 137403 (2015).

29. Huber, R. et al. How many-particle interactions develop after ultrafast excitation of an electron-hole plasma. Nature 414, 286-289 (2001).

\section{Acknowledgements}

The authors acknowledge support from Deutsche Forschungsgemeinschaft through collaborative research centre SFB 767 and the Emmy Noether programme, from the European Research Council (Advanced Grant no. 290876), the Eliteprogramm of BadenWürttemberg Stiftung and the European Commission via the Marie Curie Carrier Integration Grant.

\section{Author contributions}

A.L. and D.B. conceived the ideas and supervised the work. T.R., M.L. and M.F.S. built the laser system and fabricated the nanocircuits. V.K. numerically modelled the nanoantennas. T.R. and M.L. performed the tunnelling measurements. T.R., D.B. and A.L. wrote the manuscript. All authors contributed to the scientific discussions.

\section{References}

1. Herink, G., Solli, D. R., Gulde, M. \& Ropers, C. Field-driven photoemission from nanostructures quenches the quiver motion. Nature 483, 190-193 (2012).

2. Schertz, F., Schmelzeisen, M., Kreiter, M., Elmers, H.-J. \& Schönhense, G. Field emission of electrons generated by the near field of strongly coupled plasmons. Phys. Rev. Lett. 108, 237602 (2012).

\section{Competing financial interests}

The authors declare no competing financial interests. 


\section{Methods}

Source of control transients. Most elements of the Er:fibre laser system providing the ultrashort optical control pulses exploited in our experiment have been described previously ${ }^{17}$. For the first time, passively phase-locked 1.4-cycle near-infrared transients were generated. The repetition rate was as high as $80 \mathrm{MHz}$. Another innovative benefit of the set-up was achieved by placing the pair of fused-silica wedges before the last Er:fibre amplifier and final compression of the pulse to a duration of 6 fs. In contrast to earlier approaches based on Ti:sapphire technology, only the CEP of the output is controlled in this way, with negligible influence on the pulse envelope. Note that our control source relies on fibre-optic components provided by the telecom industry, rendering it extremely compact and robust. This fact is crucial in establishing an important step towards a future generation of solidstate nanoelectronics driven by phase-locked optical transients that operate on sub-cycle timescales in the attosecond range.

Optical characterization of control transients. To monitor the CEP we implemented an $f$-to- $2 f$ spectral interferometer ${ }^{20}$ in parallel to the main optical branch by deflecting less than $1 \mathrm{~mW}$ of average power. The fused-silica wedges had an angle of $2.9^{\circ}$ and their insertion was changed with a speed of $15 \mu \mathrm{m} \mathrm{s}^{-1}$, while recording the spectrum every $300 \mathrm{~ms}$. The position of the wedge shifted the CEP of the output pulses in agreement with a calculation of the difference between group and phase velocities in fused silica (Fig. 2b).

For a full temporal reconstruction of the pulse we performed a direct characterization via 2DSI (ref. 19). This method allowed reconstruction of the electric-field profile, that is, the amplitude and phase of the electromagnetic wave. This is particularly suited to super-octave-spanning spectra. The shear was set to a moderate value of $5 \mathrm{THz}$. By fine-tuning the dispersion via an SF10 prism pair, we obtained an almost flat phase over the entire spectrum. This result corresponds to a pulse duration of $6.3 \mathrm{fs}$ and 1.4 optical cycles at the central wavelength of $1,325 \mathrm{~nm}$.

Antenna fabrication and characterization. The nanoscale electronic circuit equipped with a plasmonic antenna was fabricated by electron-beam lithography. Spin coating was used to deposit a high-resolution positive photoresist (polymethyl methacrylate (PMMA) with 950,000 molecular weight, 950K PMMA) with a thickness of $30 \mathrm{~nm}$ on a clean fused-silica substrate, which was rotated 3,000 times per minute for $50 \mathrm{~s}$. Heating it above $100{ }^{\circ} \mathrm{C}$ before deposition evaporated residual water on the surface. The photoresist layer was annealed by soft baking at $160{ }^{\circ} \mathrm{C}$ for $3 \mathrm{~h}$. The sample was laterally patterned by exposing the photoresist to the beam of a scanning electron microscope. The accelerating voltage was set to $10 \mathrm{kV}$, the dose to $4 \mathrm{pA} \mathrm{s} \mu \mathrm{m}^{-2}$ and the step size to $3.2 \mathrm{~nm}$. The photoresist was covered by evaporation of an 8-nm-thick Al layer, which was electrically grounded to suppress charging. In the next step, the sample was treated with $0.5 \mathrm{~mol} \mathrm{NaOH}$ for $22 \mathrm{~s}$ to remove the Al layer. Methyl isobutyl ketone (MIBK), methyl ethyl ketone (MEK) and pure isopropanol at a ratio of 100:6:300 were applied for development over $20 \mathrm{~s}$. Subsequently, a $2 \mathrm{~nm} \mathrm{Cr}$ adhesion layer and $25 \mathrm{~nm} \mathrm{Au}$ were evaporated. Fast rates of $0.5 \mathrm{~nm} \mathrm{~s}^{-1}$ and $1 \mathrm{~nm} \mathrm{~s}^{-1}$ were used, respectively, to minimize the grain size of the polycrystalline Au layer. Finally, the remaining parts were lifted off in an acetone bath. Accounting for the low aspect ratio, the solvent was heated to $60^{\circ} \mathrm{C}$ and ultrasonic cleaning was applied if necessary.

The stationary electrical properties of the contacted optical antenna were studied with a sensitive source meter unit. This step was performed under vacuum conditions to avoid damage of the nanojunction. A typical result applying positive and negative bias potentials is presented in Fig. 3d. No current was detected until a voltage of $2.5 \mathrm{~V}$ was applied, corresponding to a low-bias linear resistance of the overall circuit of at least $250 \mathrm{G} \Omega$. Because the resistance of the Au leads was $\sim 1 \mathrm{k} \Omega$, the voltage dropped exclusively over the nanoscale gap. Our device safely withstands stationary voltages up to $5 \mathrm{~V}$, resulting in currents of $170 \mathrm{pA}$, equivalent to a decrease of the resistance of the tunnelling junction to $100 \mathrm{M} \Omega$. Data points were acquired by applying a triangular temporal profile centred at $0 \mathrm{~V}$ and increasing the amplitude by $0.1 \mathrm{~V}$ after each sweep, thus ensuring that there were no permanent changes to the antenna.

Numerical simulations of the field enhancement were performed using a boundary element method ${ }^{23}$. The plasmonic frequency response of the antenna was multiplied by the excitation spectrum to extract the transient field profile at the nanogap.

Modelling of the instantaneous current. To assess the time-dependent transport phenomena from our experimental data, we assumed that the current $I$ follows the electric field $E$ according to a Fowler-Nordheim behaviour, that is, $I=A E^{2} \exp (-B / E)$, where $A$ and $B$ are parameters characterizing the transport properties of a tunnelling junction, which might vary according to different biasing regimes.

When the optical field profile $E(t)$ is used, this dependence produces the instantaneous current. The measured total current is obtained as an integral over time of this quantity. We then iteratively calculate the integrated current as a function of the peak electric field when the pulse is assumed to be cosine-like. The output can be used to fit the experimental data depicted in Fig. 4b, resulting in a quantitative calibration of our estimate.

The values obtained for these dynamic conditions are $A_{\text {optical }}=1.2 \times 10^{9} \mathrm{pA} \mathrm{nm}^{2} \mathrm{~V}^{-2}$ and $B_{\text {optical }}=14.14 \mathrm{~V} \mathrm{~nm}^{-1}$. Owing to the vastly different character of the stationary case, a different parameter set of $A_{\text {static }}=896 \mathrm{pA} \mathrm{nm}^{2} \mathrm{~V}^{-2}$ and $B_{\text {static }}=0.875 \mathrm{~V} \mathrm{~nm}^{-1}$ underlies the fit shown as a dashed line in the inset of Fig. 3d. 